\title{
COMPLICADEX? NÃO! SIMPLEX!
}

\author{
COMPLICADEX? NO! SIMPLEX!
}

\author{
Gabriel Luís da Conceição * / ** / *** \\ gabrielluis_matematica@yahoo.com.br \\ * Universidade Federal Rural do Rio de Janeiro, Seropédica/RJ - Brasil \\ ** Faculdade Sul Fluminense, Volta Redonda/RJ - Brasil \\ *** Centro Universitário Geraldo di Biase,Volta Redonda/Rj - Brasil
}

\section{Resumo}

Este trabalho objetiva analisar a possibilidade de inserção da tecnologia, articulando-a com a Matemática nas aulas de Pesquisa Operacional, mais especificamente nos tópicos relativos ao método Simplex, algoritmo de solução de problemas de Programação Linear. Trata-se de um tema essencial da Matemática aplicada a diversas áreas da ciência, como Administração, Engenharias, Economia, etc, e temida por seu formato extenso de resolução, denominado por muitos de "complicadex". Norteamos nosso trabalho pela seguinte questão: como podemos explorar os conceitos e aplicações do método Simplex em sala de aula de maneira significativa aos futuros profissionais que dele farão uso? Para atingirmos os objetivos propostos realizamos um estudo experimental com alunos da graduação em Administração, onde utilizamos a metodologia de pesquisa-ação e os pressupostos da Educação Matemática. As construções e interações dos alunos na realização das atividades nos levaram a perceber que a introdução da tecnologia contribui de forma significativa para a aprendizagem desses conceitos, possibilitando a interação e consequente compreensão dos conceitos, temas e propriedades do Simplex por parte dos discentes e também possibilita uma melhora significativa na avaliação da disciplina.

Palavras-Chave: Educação Matemática; Pesquisa Operacional; Método Simplex; Tecnologia.

\begin{abstract}
This work aims to analyze the possibility of integration of technology, linking it with Mathematics in Operational Research classes, specifically on topics related to the Simplex method, linear programming problem solving algorithm. It is an essential theme of mathematics applied to various areas of science, such as Management, Engineering, Economics, etc., and feared for its extensive format resolution, called by many "complicadex". We are guided our work by the following question: how can we explore the concepts and applications of the Simplex method significantly classroom to future professionals who will use it? To achieve the goals we conducted an experimental study with undergraduate students in Administration, where we use the methodology of action research and assumptions of mathematics education. The buildings and interactions of students in performing activities led us to realize that the introduction of technology contributes significantly to the learning of these concepts, allowing interaction and consequent understanding of the concepts, themes and properties of Simplex by the students and also enables a significant improvement in the assessment of discipline.
\end{abstract}

Keywords: Mathematics Education; Operational Research; Simplex method; Technology. 


\section{INTRODUÇÃo}

A Matemática é aplicada a diversas áreas da ciência e participa efetivamente no desenvolvimento de novas teorias, no avanço da tecnologia, e no crescimento da ciência de modo geral, no entanto mesmo a afirmação acima sendo unânime, eu como professor de Matemática frequentemente observo dos alunos, inclusive os da educação superior uma aversão a ela, um obstáculo que nos preocupa, e nos leva a agir.

Em uma das disciplinas em que atuo, denominada de Pesquisa Operacional esta preocupação se acentuou, com um desempenho muito abaixo do esperado por parte dos alunos quando chegávamos em um tópico da mesma, o método Simplex, e minha formação em Educação Matemática, que se preocupa não só com a Matemática, mais com as relações entre o ensino e a aprendizagem da mesma, associando-a com a Pedagogia e a Psicologia, me fizeram partir para a ação. Buscando "novas" formas para o processo de ensino e de aprendizagem do método Simplex nas aulas de Pesquisa Operacional nos surgiu a tecnologia como possível parceira nesta empreitada.

O objetivo deste texto é discutir e analisar a possibilidade de inserção da tecnologia para auxiliar e complementar as aulas referentes ao método simplex, na cadeira de Pesquisa Operacional, para alunos da educação superior. E, para que os objetivos sejam cumpridos, buscamos responder a seguinte questão: como podemos explorar os conceitos e aplicações do método Simplex em sala de aula de maneira significativa aos futuros profissionais que dele farão uso?

Utilizaremos a metodologia de pesquisa-ação. Sobre essa metodologia, não há certeza sobre quem a utilizou pela primeira vez, embora alguns a atribuam a Lewin (1946). Trata-se de uma metodologia que investiga a própria prática a fim de aperfeiçoá-la. Talvez por isso não saibamos ao certo quando ela iniciou, pois, todo profissional de educação que se preze deve tornar a sua prática profissional uma prática de pesquisa-ação, ou seja, elaborarmos ações de forma planejada e estruturada, para que a nossa prática seja aperfeiçoada.

A pesquisa-ação é um tipo de pesquisa social que é concebida e realizada em estreita relação com uma ação ou com a resolução de um problema coletivo e no qual os pesquisadores e os participantes representativos da situação da realidade a ser investigada estão envolvidos de modo cooperativo e participativo. (THIOLLENT, 1995, p. 14)

Sobre esta metodologia, aprofundaremos no decorrer do texto.

O trabalho está estruturado em cinco seções, sendo a primeira a Introdução, o construímos da seguinte forma: 
Na seção dois, intitulada, "Pesquisa Operacional: o simplex e suas aplicações" apresentamos a Pesquisa Operacional e método Simplex como técnica de solução de problemas de Programação Linear e algumas de suas diversas aplicações.

$\mathrm{Na}$ terceira seção, que denominamos, "Tecnologia e ensino de matemática em pesquisa-ação", apresentamos as relações possíveis entre Matemática e Tecnologia, bem como os pressupostos teóricos e metodológicos que nos fundamentam.

Na seção de número quatro, "O software PHP Simplex aplicado nas aulas de Pesquisa Operacional" são descritos os caminhos da pesquisa, os sujeitos, métodos e materiais utilizados nas atividades de experimentação com alunos da graduação em Administração. Neste capítulo, também descrevemos algumas das atividades propostas e seus objetivos, bem como os comportamentos e algumas discussões na realização das atividades.

$\mathrm{Na}$ quinta e última seção deste texto, "Considerações Finais" apresentamos algumas considerações referentes à pesquisa, assim como algumas reflexões pós pesquisa.

\section{PESQUISA OPERACIONAL: O SIMPLEX E SUAS APLICAÇÕES}

A Pesquisa Operacional, ou PO, é um importante ramo da Matemática Aplicada, e pode ser definida de inúmeras formas, Colin (2007) nos diz que, em qualquer definição deste ramo da ciência, três características não podem faltar (por ordem de importância): uso de métodos matemáticos para resolver problemas, desejo constante por otimização e orientações a aplicações, ela "é tão administração quanto matemática, e um matemático terá tantas dificuldades com ela quanto um administrador que não conheça nada de matemática.” (COLIN, 2007, p. 4).

Otimização é uma frequente palavra do vocabulário dos administradores, e esta disciplina, em suma, tem por premissa a otimização de recursos, minimizando os custos e maximizando os lucros de produção, de redes de distribuição, de transportes dentre outros.

Mesmo com sua evidente aplicação na produção, na distribuição e nos transportes, de forma geral, a Pesquisa Operacional, é considerada por muitos alunos, inclusive os meus, uma das disciplinas mais difíceis dos cursos de Administração, Engenharia de Produção e outros que a contém como uma das cadeiras propostas.

A PO está sob ataque! Alunos descontentes estão jogando a disciplina no lixo nas avaliações. Professores e Administradores de fora da disciplina estão questionando sua importância e sua relevância para uma educação em administração geral. De forma 
alarmante, algumas escolas a estão retirando do grupo de cursos básicos dos programas. (CARRAWAY e CLYMAN, 1997, p. 115)

Este tratamento dado a ela, e o alto índice de notas baixas me geraram inquietações que busco amenizá-las com este estudo.

Continuando a explorar a Pesquisa Operacional, um de seus grandes temas é a Programação Linear, ou PL, uma poderosa técnica gerencial, donde o método Simplex é uma das técnicas utilizadas para solução de problemas reais.

Usuários tradicionais da PL, como as indústrias petrolífera e de aviação enxergam a técnica como uma condição fundamental para lucratividade e sobrevivência no longo prazo. Outras indústrias com aplicações menos tradicionais (como a farmacêutica, por exemplo) frequentemente encontram na técnica fontes de vantagem competitiva e duradoura.

Há um número gigantesco de aplicações, e muitas delas geraram economias na casa de centenas de milhões de dólares; algumas passaram a casa dos bilhões de dólares. (COLIN, 2007, p. 1)

Percebemos que a PL, e consequentemente o método simplex, um dos seus métodos de solução, possuem enormes aplicações nas técnicas gerenciais, e além disso, Colin (2007) ainda nos informa que "vários Prêmios Nobel de Economia tiveram boa parte de seu conteúdo relacionado com a Programação Linear", mostrando-nos além da aplicação em situações práticas a sua relevância na comunidade acadêmica de modo geral.

Desde a década de 1950 ate hoje, todas as indústrias (de agronegócios, alimentícia, automobilística, de autopeças, de aviação, bancária, de construção civil, farmacêutica, florestal, de mídia e entretenimento, mineradora, naval, de papel e celulose, petrolífera, química, de serviços de saúde, siderúrgica, de telecomunicações, varejista etc) já se beneficiaram com a técnica. Uma pesquisa contemplando as empresas da Fortune 500 indicou que $85 \%$ delas usavam ou haviam usado a Programação Linear. No Brasil, empresas como Copersucar, Correios, Eletrobrás, Petrobrás e Sadia estão entre aquelas que já aplicaram a técnica. (COLIN, 2007, p.1)

Percebemos que se tratam de técnicas matemáticas amplamente aplicadas nas empresas do nosso país e do exterior, sendo o método Simplex, como dissemos, um dos modelos de solução de um problema de PL, de forma 
que "envolve uma sequencia de cálculos repetitivos por meio dos quais é possível chegar a solução de um problema de programação linear". (MOREIRA, 2010, p. 61)

A seguir, para colocar o leitor em contato com um problema de PL, apresentamos a seguir um problema proposto na obra de Moreira (2010, p. 14).

Segue a situação problema:

Uma fabrica produz dois produtos, A e B. Cada um deles deve ser processado por duas máquinas, $\mathrm{M}_{1}$ e $\mathrm{M}_{2}$. Devido a programação de outros produtos, que também utilizam essas maquinas, a máquina $\mathrm{M}_{1}$ tem 24 horas de tempo disponível para os produtos $\mathrm{A}$ e $\mathrm{B}$, enquanto a máquina $\mathrm{M}_{2}$ tem 16 horas. Para produzir uma unidade do produto $\mathrm{A}$, gastamse 4 horas em cada uma das máquinas $\mathrm{M}_{1}$ e $\mathrm{M}_{2}$. Para produzir uma unidade do produto $\mathrm{B}$, gastam-se 6 horas na máquina $\mathrm{M}_{1}$ e 2 horas na máquina $\mathrm{M}_{2}$. Cada unidade vendida do produto A gera um lucro de $\mathrm{R} \$ 80,00$ e cada unidade do produto $\mathrm{B}$, um lucro de $\mathrm{R} \$$ 60,00. Existe uma previsão máxima de demanda para o produto $\mathrm{B}$ de 3 unidades, não havendo restrições quanto a demanda do produto A. Deseja-se saber quantas unidades de A e B devem ser produzidas, de forma a maximizar o lucro e, ao mesmo tempo, obedecer a todas as restrições.

A partir do simplex, e de outras técnicas de PL, podemos após inúmeros e extensos cálculos afirmarmos que para a situação descrita acima devem ser produzidas 3 unidades de A e 2 unidades de B para produzir o lucro máximo de $\mathrm{R} \$ 360,00$ obedecendo todas as restrições impostas pelo problema.

Este trabalho surge, após inúmeras análises sobre as dificuldades encontradas no ensino e na aprendizagem de PL, especificamente do Simplex, e propomos uma forma de contornar a situação não favorável por meio de uso dos mecanismos tecnológicos aplicados em casos reais e ao trabalho em equipe.

\section{TECNOLOGIA E ENSINO DE MATEMÁTICA EM PESQUISA-AÇAO}

Vivemos em um mundo de coisas e atividades interligadas umas as outras, e a utilização da tecnologia tem adquirido uma importância cada vez maior nos diversos setores da sociedade, inclusive nas escolas e universidades, sendo denominada por "tecnologia educacional", 
O conceito de tecnologia educacional pode ser enunciado como conjunto de procedimentos que visam "facilitar" os processos de ensino e aprendizagem com a utilização de meios e suas consequentes transformações culturais (REIS, 2009, p. 13)

E de que forma ela cabe ou se comporta nas salas de aula, especificamente nas aulas de Matemática?

Nas primeiras discussões sobre a temática, segundo Ponte (2000), houveram enormes resistências, pois ficava a indagação: qual seria o papel do professor? Substituído por máquinas? Os estudos do mesmo autor mostram o contrário, pois o papel do professor é de fundamental importância neste ambiente.

A introdução da tecnologia não tira a importância do professor no processo de ensino e de aprendizagem, pois o enfoque da tecnologia educacional não são as máquinas como objeto de estudo, mas como meio de aquisição de conhecimentos (VALENTE, 2008).

A presença isolada e desarticulada da tecnologia não é, jamais, sinal de qualidade de ensino; mal comparando, a existência de alguns aparelhos ultramodernos de tomografia e ressonância magnética em determinado hospital ou rede de saúde não expressa, por si só, a qualidade geral do serviço prestado a população. É necessário estarmos muito alertas para o risco da transformação dos computadores no bezerro de ouro a ser adorado em Educação. (CORTELLA, 1995, p.34)

Assim, o docente necessita participar de forma ativa neste processo de construção de conhecimentos, sendo um motivador, mediador e orientador, pois, "a máquina precisa do pensamento humano para se tornar auxiliar no processo de aprendizado". (RIBEIRO, 2005, p. 94).

Com a Matemática não é diferente, pois Tecnologia e Matemática sempre estiveram conectadas,

Ao longo da evolução da humanidade, Matemática e Tecnologia se desenvolveram em íntima associação, numa relação que poderíamos dizer simbiótica. A Tecnologia entendida como convergência do saber e do fazer, e a Matemática são intrínsecas a busca solidaria de sobreviver e de transcender. A geração do conhecimento matemático não pode, portanto, ser dissociada da tecnologia disponível (D’AMBROSIO, 1996, p. 2) 
Neste contexto, Canavarro (1994) nos apresenta um estudo onde aponta que a utilização da tecnologia por professores de Matemática, proporciona motivação, modernização, facilitação e mudança. O processo ocorre sempre com professores e alunos em cooperação.

Para utilizarmos as oportunidades produzidas com o uso da tecnologia em nossa pesquisa, atrelamos uma metodologia que nos dá possibilidades de agirmos de tal forma, que é a metodologia de pesquisa-ação. Esta é entendida quando há ações por parte dos envolvidos no processo de investigação, a fim de buscarmos soluções e aperfeiçoamentos para problemas coletivos. De acordo com David Tripp (2005), a pesquisa-ação é uma investigação, para qualquer processo que siga o ciclo a seguir:

Figura 1: Ciclo da Pesquisa-Ação

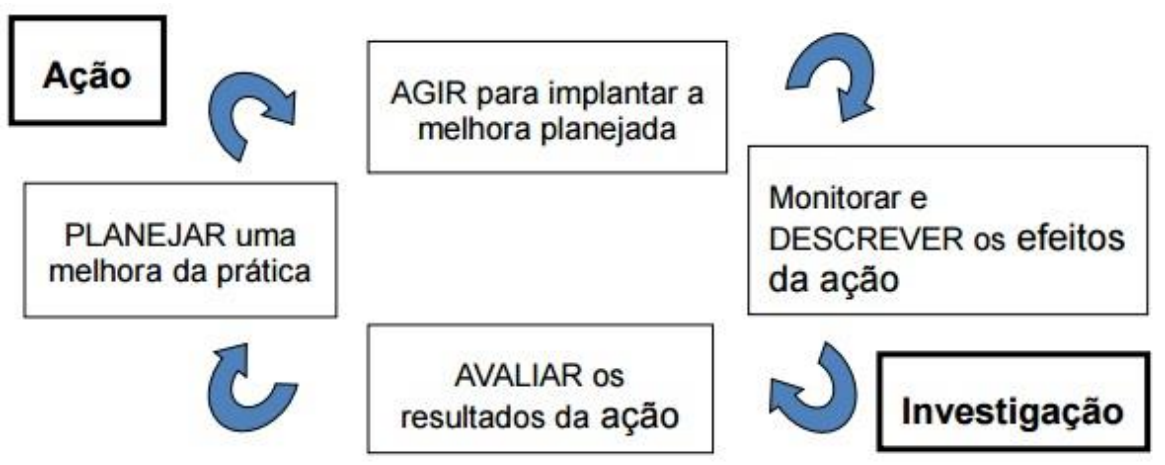

Fonte: David Tripp (2005, p. 446)

Com as fases representadas na Figura 1 é que estruturamos esta pesquisa. A saber, planejamos, implementamos, descrevemos e avaliamos todo o processo, buscando uma mudança e melhora de nossa prática.

Além das fases que regem a metodologia que escolhemos, para que ela alcance os seus objetivos é estritamente necessária a participação de todos os envolvidos em busca de um bem comum. Não se trata unicamente de um levantamento de dados, mas uma transformação de realidade, dessa forma, "é necessário definir com precisão qual ação, quais agentes, seus objetivos e obstáculos, qual exigência de conhecimento a ser produzido" (THIOLLENT, 1985, p. 16).

Escolhemos esta metodologia, pois, perante aos muitos desafios que encontramos em nossas aulas de Pesquisa Operacional, a busca por resultados qualitativos nos tem inquietado para buscarmos respostas, e o ciclo “investigação-ação-investigação" proposto por esta metodologia, nos dá a possibilidade de buscar soluções qualitativas com uma participação ativa de todos os envolvidos. 
A partir disso, buscamos investigar e agir sempre da forma mais adequada para que os conteúdos e os objetivos propostos sejam atingidos e assimilados de maneira significativa por nossos alunos, sem descuidar do rigor matemático que é exigido.

A pesquisa-ação é baseada nas ações e transformações das situações. Em todas as atividades a serem detalhadas na próxima seção deste texto utilizamos essas premissas.

\section{O SOFTWARE PHP SIMPLEX APLICADO NAS AULAS DE PESQUISA OPERACIONAL}

Nesta última seção do texto apresentaremos uma breve análise e algumas considerações acerca da utilização da tecnologia, por meio do software PHP Simplex em nossas aulas de Pesquisa Operacional, ao abordarmos o método Simplex como técnica para solucionar problemas de PL.

Esta experimentação foi realizada com alunos do quinto período do segundo semestre de 2015 do curso de bacharelado em Administração da Faculdade Sul Fluminense (FaSF), toda a turma foi envolvida no projeto. Vale ressaltar que leciono esta disciplina desde 2011 nesta instituição de ensino superior, e este foi o primeiro semestre que busquei o auxílio da tecnologia para o processo de ensino e aprendizagem do método Simplex.

Para as atividades propostas utilizamos o Laboratório de Informática da instituição, com acesso a rede mundial de computadores, bem como projetor multimídia para que cada grupo de trabalho, que denominamos GT pudesse compartilhar as suas hipóteses e conjecturas com os outros colegas, pois "uma imagem visual não apenas organiza os dados disponíveis em estruturas significativas, mas também é um fator importante na orientação do desenvolvimento analítico de uma solução". (FAINGUERLERT e NUNES, 1999, p. 55).

Os computadores conectados a internet foram amplamente utilizados para que os alunos pudessem explorar o software PHP Simplex ${ }^{1}$, ferramenta on-line para resolver problemas de Programação Linear. Seu uso é livre e gratuito. O software é capaz de resolver problemas usando o método Simplex e o método Gráfico, e não tem limitações quanto ao número de variáveis de decisão ou problemas de restrições, fato positivo, ao se comparar com outros programas similares.

1

Disponível no sitio http://www.phpsimplex.com/ 
O site é totalmente projetado para auxiliar no processo de ensino e de aprendizagem, pois não apresenta unicamente os resultados finais, mas também todas as operações intermediárias, que como vimos na primeira seção não são poucas. Ele também oferece solução direta para o uso profissional. Outras vantagens são que ele não precisa de qualquer idioma para indicar o problema, oferece uma interface clara e objetiva, sem muitos rodeios ou técnicas com as operações fáceis e intuitivas. Não é necessário instalar nada para utilizá-lo e está disponível em várias línguas, inclusive o português, muito diferente de outros softwares de PL que conhecemos, e comparando-o com o Excel, que também pode ser utilizado para resolver problemas de PL, o PHP Simplex é interativo, o que não ocorre com o Excel.

O site também contempla a parte teórica, com enunciados, conceitos chaves e um material recheado de instruções sobre a PL e sobre as suas próprias funcionalidades.

Figura 2: Homepage do PHP Simplex

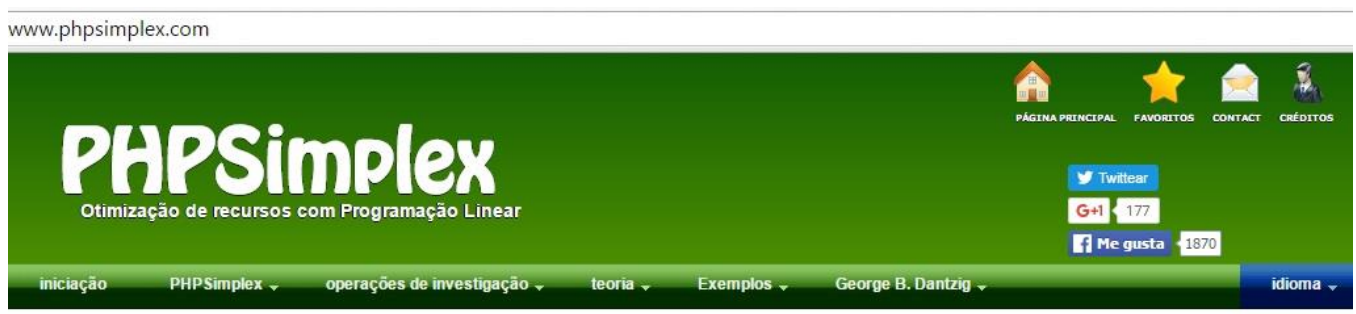

\section{PHPSimplex}

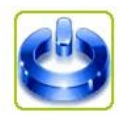

PHPSimplex é uma ferramenta online para resolver problemas de programação linear Seu uso é livre e gratuita. Para acessá-lo basta clicar no icone à esquerda, ou em "PHPSimplex" no menu uperior.

PHPSimplex é capaz de resolver problemas usando o método Simplex, o método das duas fases, eo método Figura, e não tem limitações quanto ao número de variáveis de decisão ou problemas de restrições.

Esta ferramenta é projetada para ajudar os alunos na sua aprendizagem, pois não só mostra os resultados finais, mas também as operações intermediárias. Ele também oferece solução direta para uso profissional. Outras vantagens são que ele não precisa de qualquer idioma para indicar o problema, oferece uma interface amigável, e mais perto do usuário, a operação fácil e intuitiva, que não é necessário para instalar nada de usar, e está

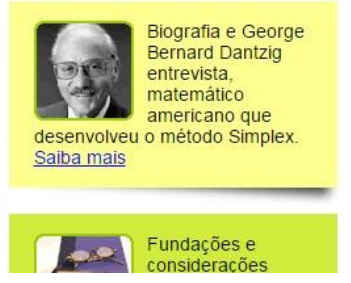

Fonte: www.phpsimplex.com

Entendemos que a tecnologia interativa do PHP Simplex como material complementar as aulas contribuiu de forma efetiva para o aprendizado, além é claro do fator motivador, pois,

Manipulando diretamente os objetos na tela do computador, e com realimentação imediata, os alunos questionam o resultado de suas ações/operações, conjecturam e testam a validade das conjecturas inicialmente através dos recursos de natureza empírica" (GRAVINA, XXX, p. 89-90) 
É também notória a importância em se trabalhar com diferentes representações, aliando o que eles constroem à mão com as ferramentas tecnológicas, de forma que ele possa realizar as atividades de diferentes formas, identificando as características e propriedades de cada etapa de construção da solução. "A habilidade de pensar em termos de diferentes tipos de sistemas de representação favorece o bom desempenho e a competência no pensar matemático". (SCHOEM FELD apud FAINGUELERNT, 1999, p. 58).

Vale ressaltar mais uma vez que a utilização da tecnologia por si só não produz a compreensão e construção do pensar matemático, para que ocorra essa compreensão e construção dos próprios conceitos é necessário desenvolvermos atividades investigativas, a fim de que o aluno assuma uma posição ativa no processo, e sinta-se no papel de um investigador, logo construtor de seu próprio conhecimento, e a matemática é um campo propício a este processo exploratório.

As atividades consistiram em duas etapas, a saber:

- Resolução problemas de PL aplicados a Administração de Empresas, de forma que cada GT recebia problemas diferentes dos demais, para que após as resoluções houvessem apresentações e consequentes debates sobre cada uma das soluções e aplicações, seja na produção, na logística, nas redes de distribuições, nos transportes, dentre outros.

- Após as formulações dos conceitos e as técnicas de resoluções bem definidas por cada grupo, nesta segunda etapa, cada GT deveria elaborar um proposta aplicada, para que outro GT pudesse resolver e após isso cada grupo apresentaria a sua solução e com isso gerar debates e discussões sobre a temática e sobre as aplicações da Pesquisa Operacional à Administração de Empresas.

Como já informamos, a turma envolvida na pesquisa foi dividida em grupos de trabalhos, com o propósito de discutir as atividades com o seu GT e com os demais, pois entendemos que a discussão é uma etapa de grande importância, e é um dos elementos essenciais para que a metodologia que escolhemos, a pesquisa-ação, seja efetivamente cumprida. 
A fase de discussão é, pois, fundamental para que os alunos, por um lado ganhem um entendimento mais rico do que significa investigar e, por outro, desenvolvam a capacidade de comunicar matematicamente e de refletir sobre o seu trabalho e o seu poder de argumentação. Podemos mesmo afirmar que, sem discussão final, se corre o risco de perder o sentido da investigação. (PONTE et al., 2003, p. 41)

A cada término de aula, cada aluno, individualmente, recebeu uma folha para as anotações, que serviram como base para as análises e considerações deste texto.

A primeira etapa das atividades, que objetivava resolver problemas reais e práticos aplicados a Administração, teve uma pequena dificuldade inicial, pois os cálculos são extensos e nem sempre as respostas coincidiam com o que o software apresentava, mas no fim foram atingidas com êxito, afinal, a esmagadora maioria dos nossos alunos tem facilidade com a tecnologia, e a utilizam para o trabalho, lazer e etc. Ao compreenderem as funcionalidades do PHP Simplex, conseguiam passear nas resoluções dos problemas e até construir hipóteses nunca presenciada por mim em turmas das quais não utilizei estas atividades.

$\mathrm{Na}$ segunda etapa, onde agora assumiram a posição de Administradores de uma empresa, e que precisavam tomar alguma decisão, o processo foi mais lento, porem não menos eficiente.

Houve um início dificultoso para formularem situações problemas em que poderiam enfrentar, mas ao final de algumas aulas, e após as minhas intervenções, conseguimos formar uma sequência de situações problemas bem interessantes, inclusive um grupo cooperando com o outro, e, passamos a investigá-las para encontrarmos a melhor solução, objetivando sempre, maximizar o lucro ou minimizar os custos.

Após a formulação das situações, houve a etapa de trocas, onde cada GT foi em busca da solução do problema elaborado por outro, e foi um sucesso, todos os problemas, uns com mais rapidez que os outros foram resolvidos com êxito. E no fim houveram as apresentações e discussões como avaliação final deste tópico, dispensando a necessidade de avaliação formal, tal como estamos acostumados, nossa famosa prova. Desta vez, ela foi realizada informalmente, em um ambiente fora da sala de aula, semelhante ao que nossos futuros administradores encontrarão em seus futuros ambientes de trabalho como profissionais da área. 
$\mathrm{O}$ ato de avaliar a aprendizagem implica em acompanhamento e reorientação permanente da aprendizagem. E, assim sendo, a avaliação exige um ritual de procedimentos, que inclui desde o estabelecimento de momentos no tempo, construção, aplicação e contestação dos resultados expressos nos instrumentos; devolução e reorientação das aprendizagens ainda não efetuadas. Para tanto, podemos nos servir de todos os instrumentos técnicos hoje disponíveis, contanto que a leitura e interpretação dos dados seja feita sob a ótica da avaliação e não de classificação. O que distingue, de fato, $o$ ato de examinar e $o$ ato de avaliar não são os instrumentos utilizados para a coleta de dados, mas sim o olhar que se tenha sobre os dados obtidos: o exame classifica e seleciona, a avaliação diagnostica e inclui. (LUCKESI, 2004, p. 4)

Como Educador Matemático, corroboro com as ideias de Luckesi, que nos apresenta o ato de avaliar. Entendo que, de fato, avaliação é muito mais do que um único exame.

Ainda no século XXI as provas traduzem a ideia de exame e não de avaliação, tal como em um passado longínquo. Também, segundo o mesmo autor, "avaliar significa subsidiar a construção do melhor resultado possível e não pura e simplesmente aprovar ou reprovar alguma coisa", a avaliação, tal como pensamos, deve construir a aprendizagem e não a engessar. E é o que nos propomos com estas atividades aliando Tecnologia e Educação Matemática.

\section{CONSIDERAÇÕES FINAIS}

Esta pesquisa que nos propomos a realizar foi desenvolvida com comprometidos alunos da educação superior, onde apresentamos a eles o método Simplex de resolução de problemas de programação linear aliando a tecnologia ao processo de ensino e de aprendizagem.

Para a fundamentação da pesquisa e concepção das atividades, utilizamos os pressupostos teóricos da Educação Matemática e da Educação, bem como pesquisadores da Matemática Aplicada, com referencias sobre a PO, a PL e o Simplex.

A metodologia da pesquisa foi a pesquisa-ação preconizada por Thiollent, nossa principal referência sobre a temática.

Na realização das atividades percebemos claramente que os alunos compreenderam as ideias, técnicas e processos do método Simplex.

As atividades foram desenvolvidas gradualmente, de forma contextualizada, permitindo uma aprendizagem significativa das ideias sugeridas. 
Ao propormos as atividades, esperávamos certa resistência por parte dos discentes, e fomos imensamente surpreendidos com a disposição e o envolvimento da maioria. Quase todas as aulas geraram frutíferas discussões, que sem dúvida acrescentaram positivamente à aprendizagem.

O desenvolvimento da proposta se deu em um ambiente fora dos pradrões convencionais da sala de aula. No entanto, sempre buscamos a utilização de uma linguagem precisa da Matemática. Além disso, as atividades foram desenvolvidas em Grupos de Trabalhos, e dessa forma possibilitou uma maior participação dos alunos, formando hipóteses e validando, ou não, suas conjecturas.

A utilização da tecnologia nos permitiu perceber uma melhor assimilação dos conteúdos contribuindo de forma efetiva na aprendizagem dos conceitos matemáticos.

Todos os momentos foram importantes, bem como todo o processo, do erro ao acerto, um desafio que conseguimos vencer juntos. O Simplex não é um desafio só para a aprendizagem, também o é para o ensino e juntos fomos melhores do que poderíamos ser sozinhos.

Verificamos que a tecnologia aliada ao ensino é uma grande parceira possível para o processo.

Podemos certamente concluir com esta pesquisa que uma proposta de ensino fora dos padrões com instrumentos que antes eram tidos como inimigos da educação, contribuem de forma efetiva para a compreensão dos conceitos, temas e propriedades da Programação Linear, em especial, do método Simplex.

\section{REFERÊNCIAS}

CANAVARRO, A. P. Concepções e práticas de professores de matemática: três estudos de caso. 1993. $361 \mathrm{f}$. Dissertação (Mestrado em Educação) - Departamento de Educação da Faculdade de Ciências, Universidade de Lisboa, Lisboa, 1994.

CARRAWAY, R. L; CLYMAN, D. R. Managerial relevance: the key to survival for OR/MS. Interfaces, v 27, n.6, pp.115-130, 1997.

COLIN, E. C. Pesquisa Operacional: 170 aplicações em estratégia, finanças, logística, produção, marketing e vendas. Rio de Janeiro: LTC, 2007.

CORTELLA, M. S. Informatofobia e Informatolatria. Equívocos na Educação. Disponível em http://www.inep.gov.br/pesquisa/bbe-online/det.asp?cod=518898type=P. Acesso em 12/07/2016. 
D’AMBROSIO, U. Educação matemática: da teoria á prática. Campinas, SP: Papirus, 1996, p. 17-28. (Coleção Perspectivas em Educação Matemática).

FAINGUELERNT, E. K. Educação Matemática: Representação e Construção em Geometria. Porto Alegre: Artes Médicas Sul, 1999.

FAINGUELERNT, E. K.; NUNES, K. R. A. Matemática: práticas pedagógicas. Porto Alegre: Penso, 2012.

GRAVINA, M. A. Geometria dinâmica: uma nova abordagem para a aprendizagem da Geometria. In: Simpósio Brasileiro de Informática na Educação, Belo Horizonte, 1996. Anais. Disponível em: http://www.ufrgs/espmat/disciplinas/geotri/pdf/maria-alice_geometriadinamica1996-vii sbie.pdf. Acesso em 23/07/2016.

LUCKESI, C. C. Considerações gerais sobre avaliação no cotidiano escolar. Aprender à Fazer. Curitiba, PR, n.36, p.4-6, 2004.

MOREIRA, D. A. Pesquisa Operacional: curso introdutório. 2 ed. São Paulo: Cengage Learning, 2010.

PONTE, J. P. Tecnologias da informação e comunicação na formação de professores: que desafios? Revista Ibero-Americana de Educação, n. 24, p. 63-90, 2000. Disponível em: http://www.rieoei.org/rie24a03.htm. Acesso em 23/07/2016.

PONTE, J. P.: BROCADO, J.; OLIVEIRA, H. Investigações matemáticas na sala de aula. Belo Horizonte: Autentica, 2003.

RIBEIRO, O. J. Educação e novas tecnologias: um olhar para além das técnicas. In: COSCARELLI, C. V.; RIBEIRO, A. E. (Org.). Letramento digital: aspectos sociais e possibilidades pedagógicas. Belo Horizonte: Atlântica, 2005, p. 86-97.

THIOLLENT, M. Metodologia da Pesquisa-ação. 5. ed. São Paulo: Cortez, 1995.

VALENTE, J. A. As tecnologias digitais e os diferentes letramentos. Revista Pátio. Porto Alegre, RS, v. 11, n. 44, nov. 2007 / jan. 2008. 
Recebido em: 10/08/2016

Aceito em: 22/09/2016

Nome: Gabriel Luís da Conceição

(c) (7)

email: gabrielluis_matematica@yahoo.com.br

Este obra está licenciada com uma Licença Creative

Commons Atribuição 4.0 Internacional. 